\title{
Science in the time of corona
}

\author{
The COVID-19 crisis could change the way we conduct our scientific lives, for better and for worse.
}

W e are currently in the midst of what could be the most profound change to our lives in generations. A large proportion of the world's population is currently under some kind of lockdown, and this is affecting both life in general and also the way we do science in particular. In China, Wuhan is starting to emerge from its draconian lockdown, but it is not yet clear how and when the rest of the world will do the same, as this depends on exactly what course the disease takes in each region and the prospects of a vaccine. But however it happens, it is worth looking at how our scientific lives may change in both the short and the long term.

In this issue, we have two articles that present alternatives to the traditional scientific conference. These were conceived long before the consequences of COVID-19 became clear, but the crisis has heightened their relevance. The Q\&A with the organizers of the second virtual palaeontology conference discusses some of the opportunities and challenges of this style of conference. As hundreds of conferences across the world that were scheduled to take place in the next few months have been cancelled, plans are being made for some to be recreated online. It would be a really positive outcome if, profiting from the experience of those who have previously organized online conferences, a substantial proportion of conferences in the future remained online, reducing the environmental cost of conferences that has been discussed many times (for example, here) but with relatively little action taken so far.

Where non-virtual conferences do still occur, because of the very real benefits of informal in-person contact, we should look for innovative ways of reducing their environmental impact while at the same time expanding their reach. The Comment from Altermatt and colleagues discusses the ABCD (all continents, balanced gender, low carbon, diverse backgrounds) format for conference sessions, which mixes in-person presentations with live remote and pre-recorded ones. This allows contributions from a culturally and geographically diverse range of speakers at reduced environmental cost, while maintaining the benefits of a live conference.
At the time of writing, @EvoEcoSeminars are about to launch a series of seminars on YouTube that attempt to replicate another staple of the academic calendar, the guest seminar, with the added benefit of expanding the reach beyond just the host institution. Editors also regularly visit institutions to discuss their journals and the research of their hosts, and conducting such visits online is something we are looking into doing both now and in the post-COVID-19 future.

Inability to travel to conferences and seminars is by no means the only effect the crisis is having on our scientific lives. Some of these changes may be welcome, such as a renewed interest in citizen science in populations that are social distancing or a potential renaissance of evidence synthesis (as discussed in last month's Editorial), while others are far more challenging. Scientists are locked out of their labs or unable to get to field sites, posing short-term problems for maintaining projects and long-term problems with lack of data. Many are having to adapt rapidly to teaching courses online, and some are adjusting their research focus to COVID-19-related topics. Scientists are also facing the same daily challenges as all other citizens, such as coping with isolation, helping the vulnerable and obtaining basic food and medical supplies.

One of the most significant such challenges is faced by scientists who are parents, who are attempting to carry on their work duties at the same time as caring for or educating their children. Indeed, this Editorial is being written in the same room as young children. We need to be very careful that this crisis does not reverse advances that have been made in diversifying the scientific workforce, as younger, poorer and female scientists are likely to shoulder a disproportionate share of the burden of these caring responsibilities. We should embrace the additional time that some researchers currently have to devote to data analysis or peer review, but we should take steps to ensure that those who do not are not penalized. These steps should include changes to how research assessments and hiring decisions are made further down the line.

The crisis has put a spotlight on certain scientific topics, such as virology and vaccine development. One particular area that has been at the forefront of policymaking has been epidemiological modelling, a topic of which many in the ecological research community have some experience. Unlike in normal times, there has not been the time for peer review and the usual back-and-forth process by which scientific consensus emerges from the literature. Instead, policy has changed rapidly over time and has differed between countries as new models have emerged and been favoured. Journals have attempted to disseminate genuine advances as quickly as possible, while at the same time some preprint servers have attempted to regulate the posting of purely in silico manuscripts. The time for a detailed analysis of what has worked and what has gone wrong will be after the immediate crisis is over. However, it is clear that an open and diverse approach is needed, in which data and code can be scrutinized by the wider community, and a diverse set of cultural and modelling approaches are brought to bear. While it is important in times of crisis to be able to discern clear policy advice from the background noise, a reliance on a closed hierarchy of advisors may potentially miss approaches that could save lives. We should therefore not let COVID-19 distract us from increasing diversity and inclusion in science.

At Nature Ecology \& Evolution, we understand that priorities have changed drastically and that we all need to try to get through this together by being as understanding and flexible as possible. We therefore completely understand if commitments you have made to the journal need to be altered or abandoned. If possible, we just ask that you keep us updated of any changes. We also ask that authors remain patient as we try to juggle our own altered work circumstances, the possible reduction in reviewer availability, and the need to prioritize certain topics. We will do our best to take a pragmatic approach in our requests to both authors and reviewers, and will try to keep the system running as smoothly as possible.

Published online: 24 April 2020 https://doi.org/10.1038/s41559-020-1205-7 